\title{
Microwave Pyrolysis of Biomass within a Liquid Medium
}

B.J Shepherd ${ }^{\mathrm{a}}$, J. Ryan ${ }^{\mathrm{a}}$, M. Adam ${ }^{\mathrm{a}}$, D. Beneroso Vallejo ${ }^{\mathrm{a}}$, P. Castaño ${ }^{\mathrm{b}}$, E.T. Kostas ${ }^{\mathrm{a}}$, J.P. Robinson $^{\mathrm{a} *}$

${ }^{\text {a}}$ Faculty of Engineering, University of Nottingham, Nottingham. NG7 2RD. UK.

${ }^{b}$ Department of Chemical Engineering, University of the Basque Country (UPV/EHU), PO Box 644, 48080. Bilbao, Spain.

*Corresponding author

Email: john.robinson@nottingham.ac.uk

Tel: +44 (0) 1159514092 


\begin{abstract}
A new approach to pyrolysis is demonstrated that uses microwave heating combined with an external liquid media at atmospheric pressure. The liquid acts as the inerting medium instead of the traditional inert gas, and also acts as a heat-sink to maintain the external temperature at the normal boiling point of the liquid. The ability to regulate the external temperature using a liquid offers significant advantages over established pyrolysis technologies and is only possible due to the selective and volumetric heating that occurs with microwaves. The new concept overcomes many of the challenges encountered in traditional and gas-based microwave pyrolysis processes, producing a bio-oil that naturally partitions into a sugar-rich aqueous phase and a phenol-rich organic phase. Energy requirements are as low as 2 $\mathrm{kJ} / \mathrm{g}$ for $50 \%$ volatilisation, comparable to microwave pyrolysis using inert gases. It is shown that the new concept works effectively with both microwave-transparent and microwave-absorbent solvents. The liquid media also acts to eliminate arcing and prevent carbonaceous residues from forming, phenomena which have so far proved challenging for the scale-up of microwave pyrolysis processes.
\end{abstract}

KEYWORDS: MICROWAVE PYROLYSIS, SYCAMORE, LIQUID INERTED MEDIUM, BIO-OIL. 


\section{INTRODUCTION}

Pyrolysis is a technology that has significant potential within a bio-refinery as it allows transformation of sustainable, lignocellulosic biomass feedstocks into fuels and chemicals by heating them to 400$600^{\circ} \mathrm{C}$ in the absence of oxygen. The liquid product contains a wide spectrum of compounds derived from cellulose, hemi-cellulose and lignin, and this can be used as a blending component or directly as a fuel, or as a source of platform and speciality chemicals [1,2]. Limitations with current pyrolysis processes have so far prevented widespread uptake of the technology. The inherent low thermal conductivity of biomass means that conduction is the rate-determining step, and heating times can be of the order of several minutes. Fast or flash pyrolysis, processes that maximise the production of liquid bio-oil require heating times of the order of seconds. Processing biomass at these shorter timescales therefore requires a size reduction step, shredding or milling the biomass feedstock to $\sim 1 \mathrm{~mm}$ to increase the surface area to volume ratio and reduce the heating time However, milled biomass is more likely to entrain with the hot inert gas flow, and typically requires the use of several cyclones to separate effectively from the product stream [3]. The primary bio-oil products produced during pyrolysis are typically high molecular weight compounds from the depolymerisation of cellulose, hemi-cellulose and lignin. Examples include levoglucosan, 5-hydroxymethylfurfural (HMF) and isoeugenol. During conventional pyrolysis processes the outer surface of the biomass particle is treated first, as this attains the decomposition temperature before the centre of the particle. As the centre of the particle heats and decomposes, the primary products pass through the outer layer of treated biomass. This treated layer is rich in char, which acts to catalyse further decomposition of the primary pyrolysis products into a lower quality oil product, with lower intrinsic value.

\section{Opportunities for microwave pyrolysis}

Microwave heating is volumetric, so the heating rate is a function of the electric field intensity and dielectric loss factor [4] rather than thermal conductivity. This leads to a number of advantages and opportunities for microwave heating: 
- Process Flowsheet. When thermal conductivity no longer dominates then the heating rate for larger particles will be comparable to that for smaller particles, meaning that there is no need to grind the biomass prior to processing, and consequently no need for extensive cyclone systems to separate fines from the product. As the heat within the biomass is generated volumetrically there is no explicit need for a hot inert gas, and therefore the inert gas heating, condensation and separation steps can be eliminated or simplified.

- Product Quality. When biomass particles are heated volumetrically their internal temperature is higher than the surrounding environment. This creates an 'inverse' temperature gradient, with the centre of the particle becoming hotter than the outer surface, and is a phenomenon that is unique to electromagnetic heating. With microwave heating the pyrolysis process initiates at the centre of the particle, meaning that the primary decomposition products are transported through non-pyrolysed biomass and immediately quenched in a cold environment. This is depicted in Figure 1, along with the corresponding case for conventional pyrolysis. The lack of char at the outer edges means that there is less likelihood of secondary product decomposition in this case.

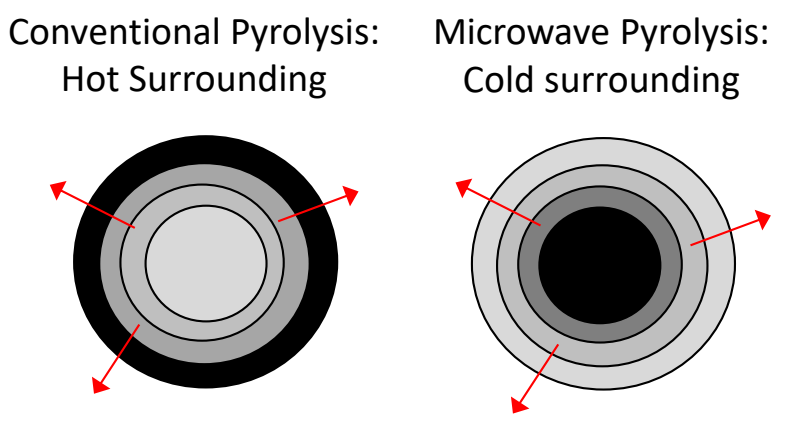

Figure 1 - Representation of thermal gradients within biomass during microwave and conventional pyrolysis. Darker areas represent higher temperatures and the presence of char. Arrows represent product mass transfer.

\section{Current status of microwave pyrolysis}

The majority of scientific literature is based on the use of low power domestic microwave ovens, which do not support a well-defined electric field, and yield a power density of around $10^{6} \mathrm{~W} / \mathrm{m}^{3}$ - below the value required to reliably achieve pyrolysis of lignocellulosic materials [5]. Carbon-based susceptor 
materials have typically been added to induce pyrolysis within these systems [6,7], where the susceptor is a much stronger microwave absorber than the biomass. The susceptor particles heat selectively over the surrounding biomass and become hotter, then subsequently transfer heat by conduction to the adjoining biomass particles. However, the use of susceptors means that the biomass is heated primarily by conduction rather than volumetrically, and this in turn means that the inherent advantages of microwave heating are lost. It is therefore not possible to realise the benefits of microwave pyrolysis by using susceptors or low-power ovens. The use of equipment with a well-defined electric field distribution that yields power densities of the order of $10^{8} \mathrm{~W} / \mathrm{m}^{3}$ can induce the fast pyrolysis of biomass without using added microwave susceptors [5], and this approach will allow the heating to be fully volumetric and subsequently allow the inherent advantages of microwave pyrolysis to be exploited [8].

\section{Challenges and barriers to scale-up}

Despite the potential opportunities to simplify the process flowsheet and improve product quality there are a number of challenges that need to be overcome if scale-up and commercial operation is to be realised. The requirement for a high power density necessitates the use of high intensity electric fields, which in many cases can exceed the breakdown voltage of the inert gas and cause arcing and subsequent damage to the reactor and microwave components. Heating heterogeneity can also occur as the dielectric properties of the resulting char cause a reduction in the penetration depth of the microwaves and localised overheating. As a result, the pyrolysis process is less controlled and poorer-quality biooil is obtained $[8,9]$. Fluidisation has been proposed as a technique to improve the heating homogeneity through the continuous movement of the biomass particles between the heating zones, however the use of a fluidising gas introduces the need for gas compression, separation and recirculation within the process flowsheet and adds significantly to the complexity and capital cost $[10,11]$. A processing concept is therefore needed that combines the high electric field intensities with inherent temperature control

\section{New Processing Concept: Liquid Inert Phase}

A novel processing concept is proposed that substitutes the inert gas for a liquid, and this concept has been developed and reported for the first time within this paper. Previous studies have used liquids in 
conjunction with a pyrolysis process, for example hydrothermal gasification [12] and solvothermal processes utilising ionic liquids [13]. In these cases, the liquids are used to transfer energy to the biomass, and thus are at temperatures of the order of $400^{\circ} \mathrm{C}$. Other studies have used liquids with microwave heating to aid wood-liquefaction processes $[14,15]$, however these systems are characterised by the use of elevated pressures to maintain the liquid phase. The concept reported in this paper is unique as it uses a liquid at atmospheric pressure to act as a heat-sink, conveying heat away from the biomass rather than providing heat to it. If a liquid is used as the inert media then the biomass can still be heated selectively provided that the dielectric loss factor is low, and this is the case for the majority of non-polar hydrocarbons. n-hexane is chosen as an example of such a liquid, and its properties are detailed in Table 1, along with nitrogen.

\begin{tabular}{|l|c|c|}
\hline \multirow{2}{*}{} & \multicolumn{2}{|c|}{ Inert Media } \\
\cline { 2 - 3 } & Gas (nitrogen) & Liquid (n-hexane) \\
\hline $\begin{array}{l}\text { Dielectric Loss Factor at } 2.45 \\
\mathrm{GHz}[4,16]\end{array}$ & $<0.001$ & 42 \\
\hline $\begin{array}{l}\text { Breakdown Voltage at 1 atm } \\
(\mathrm{kV} / \mathrm{mm})[17]\end{array}$ & 3 & 68 \\
\hline $\begin{array}{l}\text { Maximum Temperature at 1 } \\
\text { atm }\left({ }^{\circ} \mathrm{C}\right)\end{array}$ & $>500$ & 0.126 \\
\hline $\begin{array}{l}\text { Thermal conductivity }(\mathrm{W} / \mathrm{m} . \mathrm{K}) \\
{[17]}\end{array}$ & 0.026 & 25.2 \\
\hline $\begin{array}{l}\text { Convective heat transfer } \\
\text { coefficient W/m }{ }^{2} . \mathrm{K} \text {. }\end{array}$ & 5.2 & \\
\hline
\end{tabular}

Table 1 - Physical and system properties of gaseous and liquid inert media for microwave pyrolysis.

Comparative heat transfer coefficients calculated based on $\mathrm{Nu}=2$ and a spherical biomass particle with $10 \mathrm{~mm}$ diameter [18]. All properties quoted at $20^{\circ} \mathrm{C}$, unless otherwise stated.

When a liquid is used the convective heat transfer coefficient is around five times higher, meaning that there will be greater heat loss from biomass to a liquid than to a gas. However, the maximum temperature that can be reached within the liquid is its normal boiling point, which in the case of $n$ hexane is $68^{\circ} \mathrm{C}$. Further heat transfer results in boiling of the liquid, so the liquid needs to be present in excess or refluxed back into the pyrolysis vessel. The bulk temperature of the pyrolysis system is therefore significantly lower, and potentially more controllable when a liquid media is used. Table 1 shows that the breakdown voltage for $n$-hexane is over ten times higher than that of nitrogen at 
equivalent conditions. This means that high intensity electric fields can be utilised safely, without arcing, to give power densities of $10^{7}-10^{8} \mathrm{~W} / \mathrm{m}^{3}$ needed to achieve pyrolysis.

The focus of this study is to use theoretical and experimental approaches to validate this new concept and establish the extent to which woody biomass can be pyrolysed. The specific objectives are:

- Estimate the temperature distribution within the biomass particle when immersed within a liquid, and how this varies with particle size and microwave power density

- Determine the extent to which pyrolysis can be achieved when the biomass is immersed within a liquid, and the corresponding energy requirements

- Provide a preliminary indication of the quality of the obtained liquid products 


\section{METHODOLOGY}

\subsection{Microwave Experimental System}

Sycamore (Acer pseudoplatanus) was used in the microwave pyrolysis process as a model feedstock.

Blocks were cut to $45 \times 15 \times 15 \mathrm{~mm}$, with an average mass of $5-6 \mathrm{~g}$. The moisture content was $6.4 \%$ (ASTM D4442). n-hexane (Reagent grade) was supplied by Fisher Scientific. Microwave pyrolysis experiments were carried out using a $0-2 \mathrm{~kW}$ microwave generator operating at $2.45 \mathrm{GHz}$ and a single mode cavity. An automatic three stub tuner was used to maximise the absorbed power and to $\log$ forward and reflected power. The sycamore sample was placed within a $25 \mathrm{~mm}$ diameter Pyrex tube, and covered with $100 \mathrm{~mL}$ n-hexane. A microwave-transparent ceramic block was placed on top of the sycamore block to reduce buoyancy and ensure the sample was contained within the waveguide section where the electric field intensity is highest. The upper section of the tube was contained within an electromagnetic choke and connected to a condenser. Vaporised hexane was condensed and allowed to reflux back into the reactor to provide a constant level of liquid over the biomass. The waveguide and choke were purged with nitrogen at $10 \mathrm{~L} / \mathrm{min}$ to provide a secondary inert atmosphere to prevent combustion of the solvent in case of failure of the tube. A schematic of the experimental system is shown in Figure 2. 


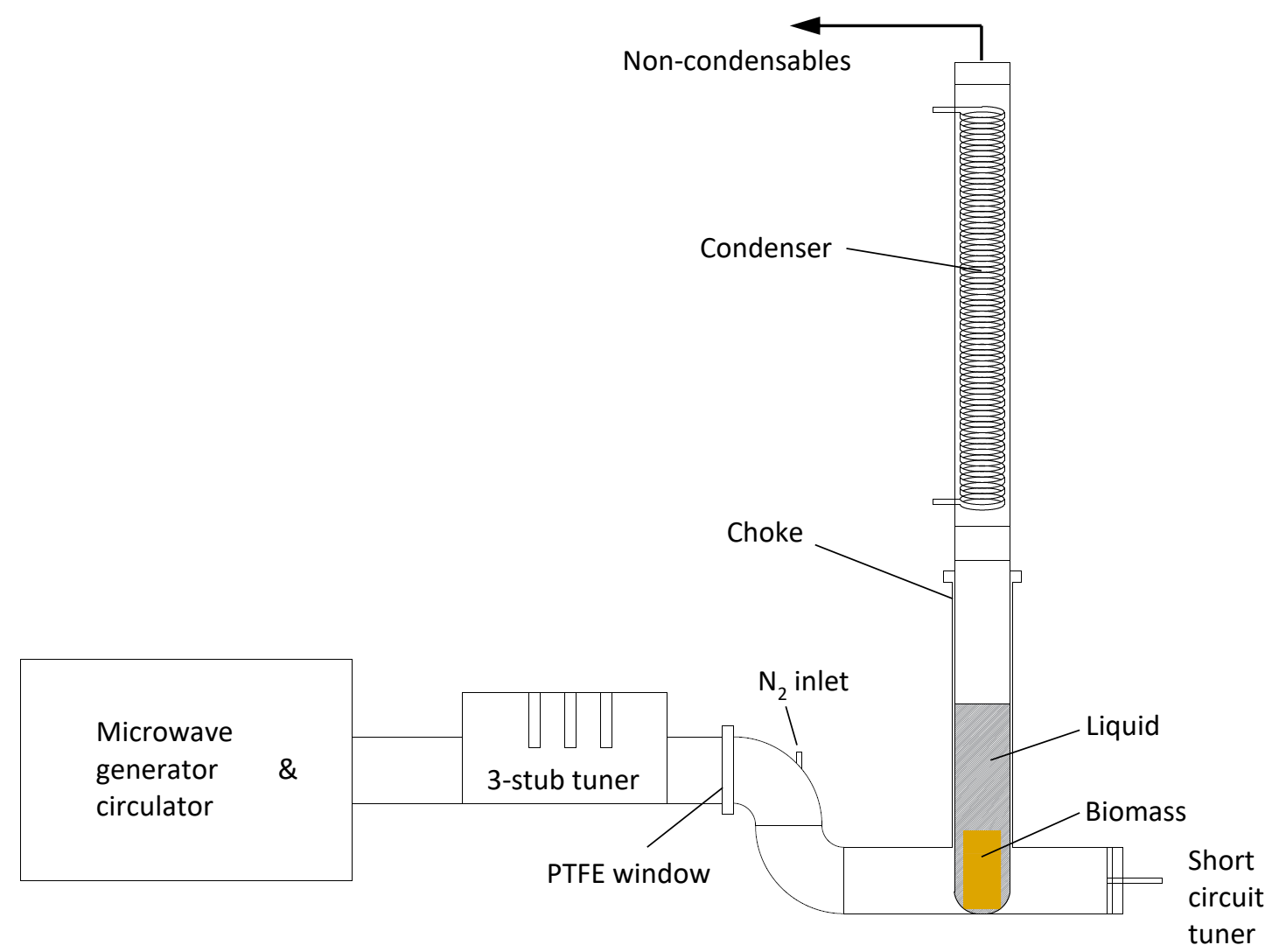

Figure 2 - Liquid-inerted microwave pyrolysis system

Experiments were carried out at $1-2 \mathrm{~kW}$ for $10-30$ seconds, to give an energy input range of $0.6-3.8$ $\mathrm{kJ} / \mathrm{g}$. After the pyrolysis process the remaining biomass sample was washed with acetone and dried at $105^{\circ} \mathrm{C}$ until a constant mass was obtained. Pyrolysed samples were then weighed and percentage mass losses were calculated.

\subsection{Heat Transfer Simulations}

It is not possible to physically measure temperature within a biomass block during microwave heating. Instead, the temperature distribution within the heated biomass was estimated using numerical simulations of volumetric heating and simultaneous conductive/convective heat transfer within a system with comparable geometry to that used in pyrolysis experiments. Such simulations can be used to determine the minimum particle size and microwave power density needed to achieve pyrolysis temperatures, parameters that can subsequently be investigated experimentally. The penetration depth of woody biomass at room temperature and $2.45 \mathrm{GHz}$ is of the order of $30 \mathrm{~cm}$ [5], over six times greater than the particle dimension considered in this study. Thus, for the purposes of the simulations the 
electric field intensity and resulting power density were considered to be uniform throughout the biomass particle. Convective heat transfer was assumed to take place from the outer surface of the particle to the liquid, with natural convection assumed with a Nusselt Number $(\mathrm{Nu})=2$. Conductive heat transfer occurred within the biomass particle, with thermal conductivity taken as a constant equal to $0.155 \mathrm{~W} / \mathrm{m} . \mathrm{K}$ [10]. Spherical particles were considered due to their symmetry, and also due to established empirical heat transfer correlations for spheres. For spherical particles a finite element method was used to establish the temperature distribution with time; the particles were meshed into 64512 triangles and 32513 nodes. The temperature of each element was established over an incremental increase in time by considering the net energy absorbed through volumetric heating and the heat gained/lost by conduction from adjacent elements. Rectangular blocks were also studied to provide a direct comparison with the experimental system, and in this case the particle was meshed into 112640 triangles and 56769 nodes. The heat capacity of the biomass and heat transfer to the liquid varies with temperature, and the relationship given by Adam et al. [10] was used for the numerical simulations, with an average density of $560 \mathrm{~kg} / \mathrm{m}^{3}$. It was assumed that the biomass particle was completely immersed within the liquid, and the liquid temperature was assumed to be constant at the normal boiling point throughout the heating process.

\subsection{Thermogravimetric Analysis (TGA) of Pyrolysed Sycamore Blocks}

A pyrolysed block was analysed to gain an understanding of the internal thermochemical decomposition induced by the inerted liquid pyrolysis process. The pyrolysed block was cut along its longitudinal axis in order to inspect the condition of the internal core. Samples $(10-15 \mathrm{mg})$ were taken from the internal core and analysed by TGA using the method outlined in Lester et al. [19]. A sample of non-pyrolysed sycamore (10-15 mg) was also included in order to identify difference in thermal degradation profiling.

\subsection{Analysis of liquid product}

Preliminary characterisation of the organic and aqueous product phases was carried out using a Shimadzu GC-2010 coupled to a TRB-1-MS column (50 m and $0.15 \mathrm{~mm}$ i.d.) and a Shimadzu QP2010S mass spectrometer detector. The operating conditions were as follows: a split ratio of 50:1 $(0.02 \mu \mathrm{L}$ of oil sample in $1 \mu \mathrm{L}$ of dichloromethane) and an injector temperature of $180^{\circ} \mathrm{C}$; a column temperature of 
$45^{\circ} \mathrm{C}$ for $2 \mathrm{~min}$, which was then heated up to $300{ }^{\circ} \mathrm{C}$ at $8{ }^{\circ} \mathrm{C} \mathrm{min}{ }^{-1}$, holding this temperature during 10 min. A helium flowrate of $1.15 \mathrm{~mL} \mathrm{m^{-1 }}$ was used. The mass spectra and retention data were used to identify the compounds by comparing them with those of the standard in the NIST 140 Mass Spectral Database. Samples were dehydrated prior to GCMS analysis. Water content was measured using a CA200 Coulometric Karl Fisher moisture meter.

\section{RESULTS \& DISCUSSION}

\subsection{Temperature Simulations}

\subsubsection{Power density and particle size requirements for spherical particles}

A target temperature of $400^{\circ} \mathrm{C}$ was set for the centre of the biomass particle, this being the temperature at which pyrolysis is achieved. The surrounding n-hexane was assumed to be at its boiling point at atmospheric pressure $\left(68^{\circ} \mathrm{C}\right)$. Thermal equilibrium is achieved when the power absorbed volumetrically from microwave heating equals the power dissipated into the liquid through conventional heat transfer. With a target temperature of $400^{\circ} \mathrm{C}$, a threshold power density and particle diameter can be identified as those that result in thermal equilibrium. Under this condition, i.e. when the internal temperature is $400^{\circ} \mathrm{C}$ the rate of heat conduction through the biomass is equal to the rate of convection from the particle surface to the surrounding liquid, with the particle surface temperature being higher than the surrounding liquid but less than the internal temperature of $400^{\circ} \mathrm{C}$. Finally, at equilibrium the rate of heat transfer by conduction and convection equal the rate of volumetric heating due to dissipation of microwave energy to heat. Figure 4 shows particle diameter and power density needed to achieve thermal equilibrium at $400^{\circ} \mathrm{C}$, as well as the corresponding biomass surface temperature in each case. 


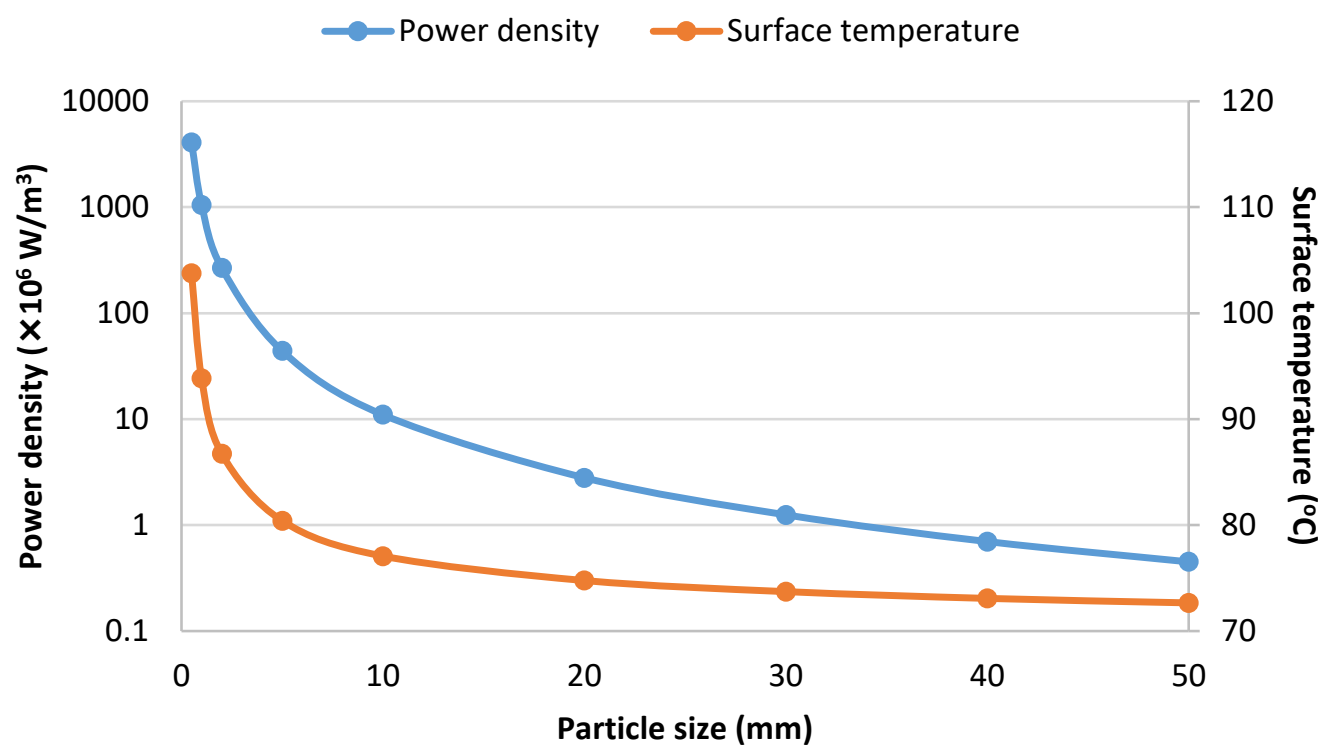

Figure 3 - Minimum power density required to achieve thermal equilibrium, and resultant particle surface temperature for spherical particles. $\mathrm{T}=400^{\circ} \mathrm{C}$ at the centre of the particle.

From Figure 3, the power density required to achieve thermal equilibrium at $400^{\circ} \mathrm{C}$ increases exponentially as particle size decreases. With smaller particles the surface area to volume ratio is larger, leading to a high heat flux from the particle surface to the surrounding liquid and a corresponding need for higher rates of volumetric heating in order to attain $400^{\circ} \mathrm{C}$ at the particle core. The maximum power density that can be achieved in a domestic-type microwave oven is of the order of $10^{5} \mathrm{~W} / \mathrm{m}^{3}$, so equipment of this type will not be sufficient to induce pyrolysis within n-hexane, even with particle sizes of $50 \mathrm{~mm}$. Power densities of the order of $10^{7}-10^{8} \mathrm{~W} / \mathrm{m}^{3}$ can be achieved in single-mode microwave heating systems, which according to Figure 3 will be sufficient to achieve temperatures of $400^{\circ} \mathrm{C}$ at the centre of any biomass particles larger than $5-10 \mathrm{~mm}$. The surface temperature of the biomass particle also varies exponentially with particle size. Thermal equilibrium is achieved with surface temperatures from $73-103^{\circ} \mathrm{C}$. The critical heat flux for n-hexane has been reported to occur when the surface temperature is $120^{\circ} \mathrm{C}$ [20]. As the surface temperatures predicted in Figure 4 are below this value this indicates that nucleate boiling will occur during the microwave pyrolysis process, and the assumption of natural convection with $\mathrm{Nu}=2$ is valid. 


\subsubsection{Heating time and temperature distribution for spherical particles}

Figure 3 shows that temperatures high enough to achieve pyrolysis can be achieved when single mode or high-power microwave heating devices are used, however the surface temperature is significantly below $400^{\circ} \mathrm{C}$. The simulations suggest that the centre of the biomass particle will be pyrolysed, whereas the outer regions will remain untreated. The temperature distribution throughout the particle was investigated using numerical simulations to assess the extent to which pyrolysis is likely to be achieved, and the amount of 'non-pyrolysed' biomass that remains at the surface due to the presence of the liquid at its boiling point. In this case a non-equilibrium simulation was carried out with a fixed power density $\left(5 \times 10^{7} \mathrm{~W} / \mathrm{m}^{3}\right)$, and the temperature established throughout the particle at varying heating times $(\mathrm{t})$. Figure 5 shows the predicted temperature distribution with time for a $10 \mathrm{~mm}$ spherical biomass particle.

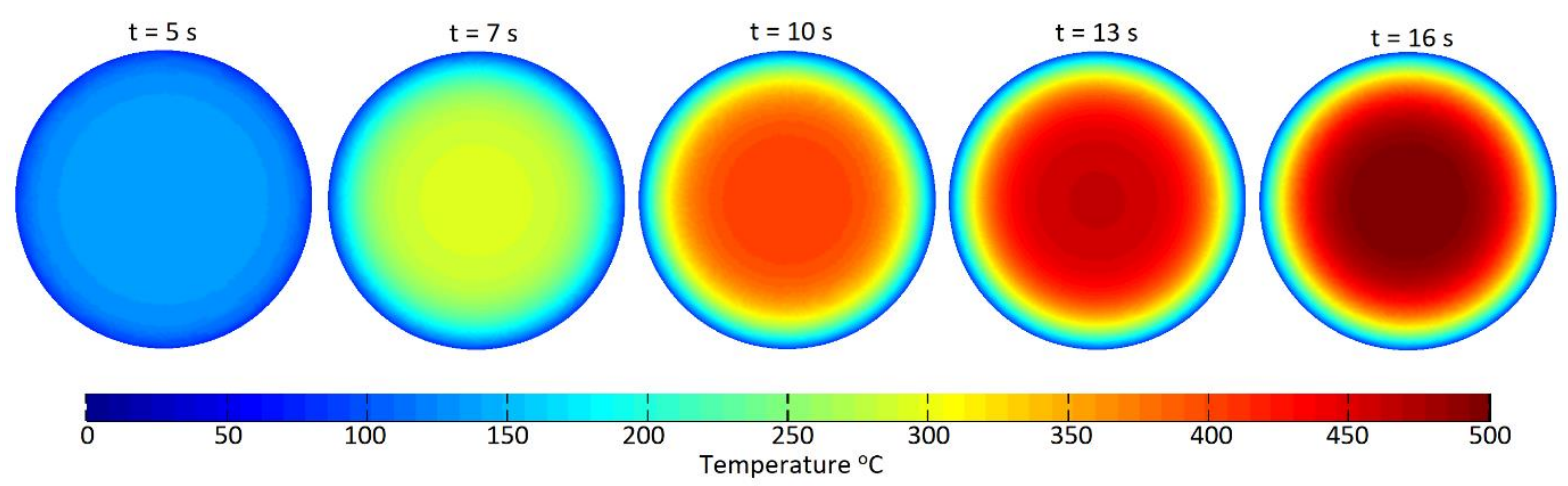

Figure 4 - Temperature gradient within a $10 \mathrm{~mm}$ biomass particle with a power density of $5 \times 10^{7} \mathrm{~W} / \mathrm{m}^{3}$ and different heating times.

As heating time increases the internal temperature increases, whilst the surface temperature remains close to the n-hexane boiling point of $68^{\circ} \mathrm{C}$. When the power density is $5 \times 10^{7} \mathrm{~W} / \mathrm{m}^{3}$ a heating time of around 10 seconds is required to achieve an internal temperature of $400^{\circ} \mathrm{C}$, whilst heating for 16 seconds increases the internal temperature to $500^{\circ} \mathrm{C}$. From Figure 3, a power density of $5 \times 10^{7} \mathrm{~W} / \mathrm{m}^{3}$ is higher than the threshold value required to achieve thermal equilibrium for a $10 \mathrm{~mm}$ particle, so it is to be expected that the core temperature will increase beyond $400^{\circ} \mathrm{C}$ under these conditions. Beyond 10 seconds there does not appear to be any significant change to the temperature within the outer $2 \mathrm{~mm}$ of the particle, whereas the core temperature increases further. The outer 'layer' within $2 \mathrm{~mm}$ of the surface 
will therefore not be pyrolysed when using $\mathrm{n}$-hexane as the inerting media, thus restricting the bio-oil yield that can be obtained. Figure 4 suggests that the possible bio-oil yield will be relatively low for small particles, where the $2 \mathrm{~mm}$ layer comprises the majority of the particle mass but could be much higher when larger particle diameters are used.

\subsubsection{Rectangular particles (Sycamore Block Simulation)}

Spherical particles present a convenient system to explore the competing physical effects and the resultant temperatures, as their inherent symmetry reduces the computational complexity of the simulations. This approach allows a large number and range of parameters to be investigated, however it is not possible to validate the output of the simulations with an experimental system due to the simplified geometry. The simulations shown in Figure 3 and Figure 4 suggest that a power density of 5 $\mathrm{x} 10^{7} \mathrm{~W} / \mathrm{m}^{3}$ and a heating time of 16 seconds will cause the internal core of the biomass to be pyrolysed, with an outer layer of $2 \mathrm{~mm}$ remaining non-pyrolysed. The temperature profile within a rectangular block with dimensions of that used in the microwave system $(45 \times 15 \times 15 \mathrm{~mm})$ was simulated, with a power density of $5 \times 10^{7} \mathrm{~W} / \mathrm{m}^{3}$, a heating time of 16 seconds and in $\mathrm{n}$-hexane as a surrounding medium. The results of the simulation are shown in Figure 5.

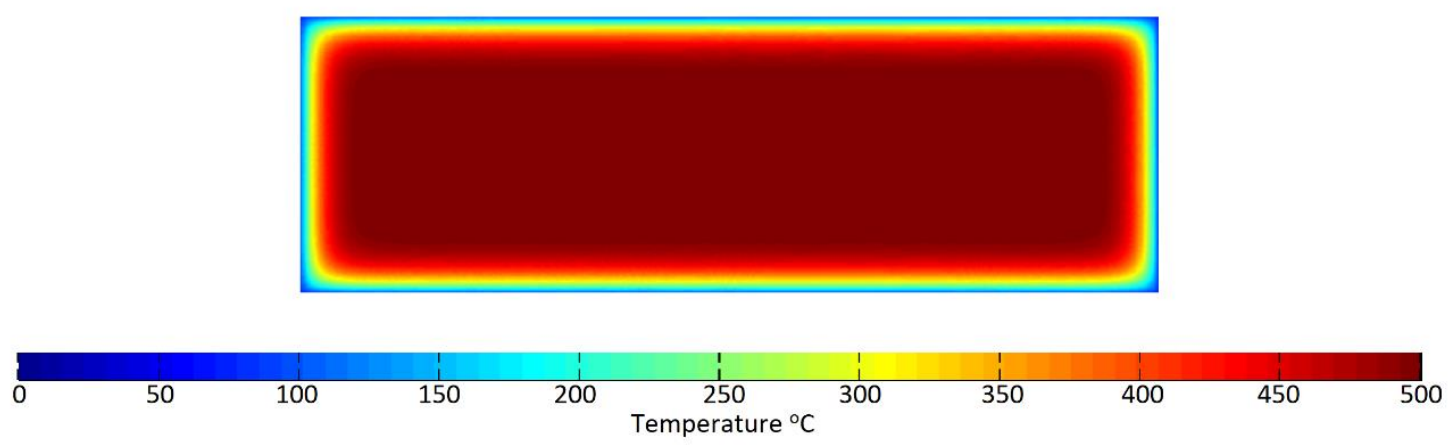

Figure 5 - Temperature gradients within a $45 \times 15 \times 15 \mathrm{~mm}$ sycamore block with a power density of $5 \times 10^{7} \mathrm{~W} / \mathrm{m}^{3}$ at 16 seconds.

As with the spherical particles, the core of the biomass block reaches $>500^{\circ} \mathrm{C}$ whereas the outer layer of around $2 \mathrm{~mm}$ in depth does not achieve a temperature greater than $300^{\circ} \mathrm{C}$. The similarity in temperature distribution and heating time between the different geometries is due to thermal conductivity being the rate-determining step for heat transfer within biomass. The convective heat flux 
is influenced by the particle shape and surface geometry, however as convection is not the ratedetermining step then changes to convective heat flux has a negligible influence on the overall temperature distribution. As well as providing a direct comparison with experimental data, Figure 4 also depicts that the computationally-simpler simulations using spherical particles offer a suitable approximation to a more realistic but computationally-intensive geometry.

\subsection{Pyrolysis Experiments}

\subsubsection{Validation of temperature simulations}

An example of the cross-section of a sycamore block following pyrolysis within $\mathrm{n}$-hexane at $1.5 \mathrm{~kW}$ forwarded power and 12 seconds is shown in Figure 7.

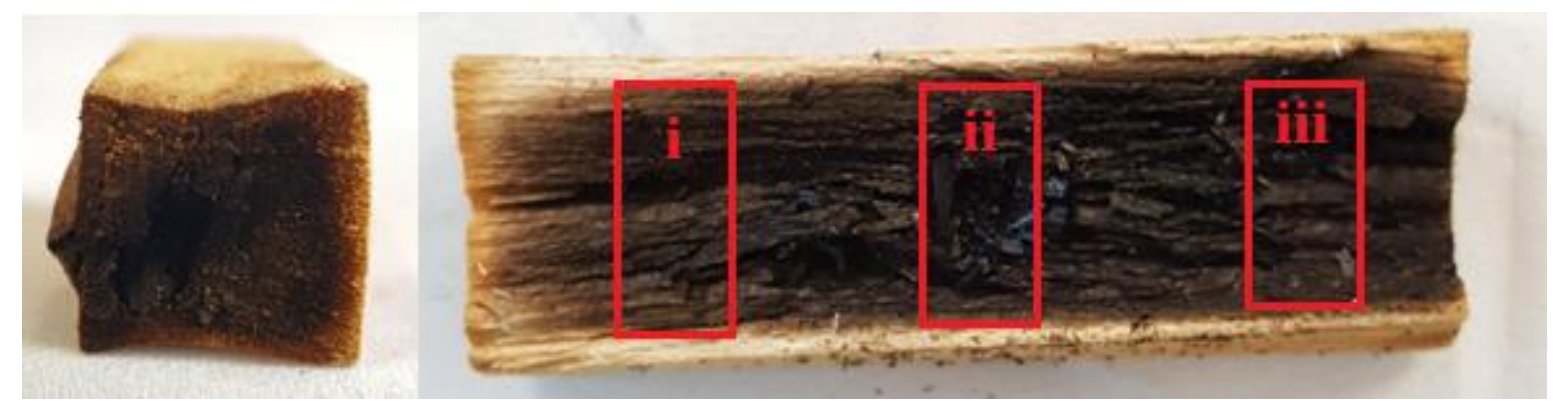

Figure 6 - Cross-sectional images of sycamore blocks after pyrolysis within n-hexane ( 45 x 15 x 15 $\mathrm{mm})$.

Figure 6 shows that the centre of the sycamore block is charred, whilst the outer edges parallel to the grain remain untreated. The cross-section correlates with the simulated temperature distribution in Figure 5, with the same outer layer of untreated material visible in both simulation and experiment. Evidently, there appears to be an effect of the grain direction which is likely to influence the heat transfer coefficient, and this will need to be investigated further. It is clear from Figure 6 that temperatures high enough to pyrolyse biomass can be achieved within the core of the block, despite the surrounding liquid medium environment not exceeding temperatures above $68^{\circ} \mathrm{C}$. It is also visually evident from Figure 6 that there are varying degrees of pyrolysed sycamore within the block, with section (i) appearing to have undergone the lowest degree of pyrolysis compared to sections (ii) and (iii). It is not possible to directly measure temperatures that have been reached within the biomass block during heating. In order 
to gain an inference to temperature and an understanding of the degree of pyrolysis within the block of sycamore, TGA was performed on the three internal sections as highlighted in Figure 6 (i, ii and iii) and their thermal profiles can be seen in Figure 7
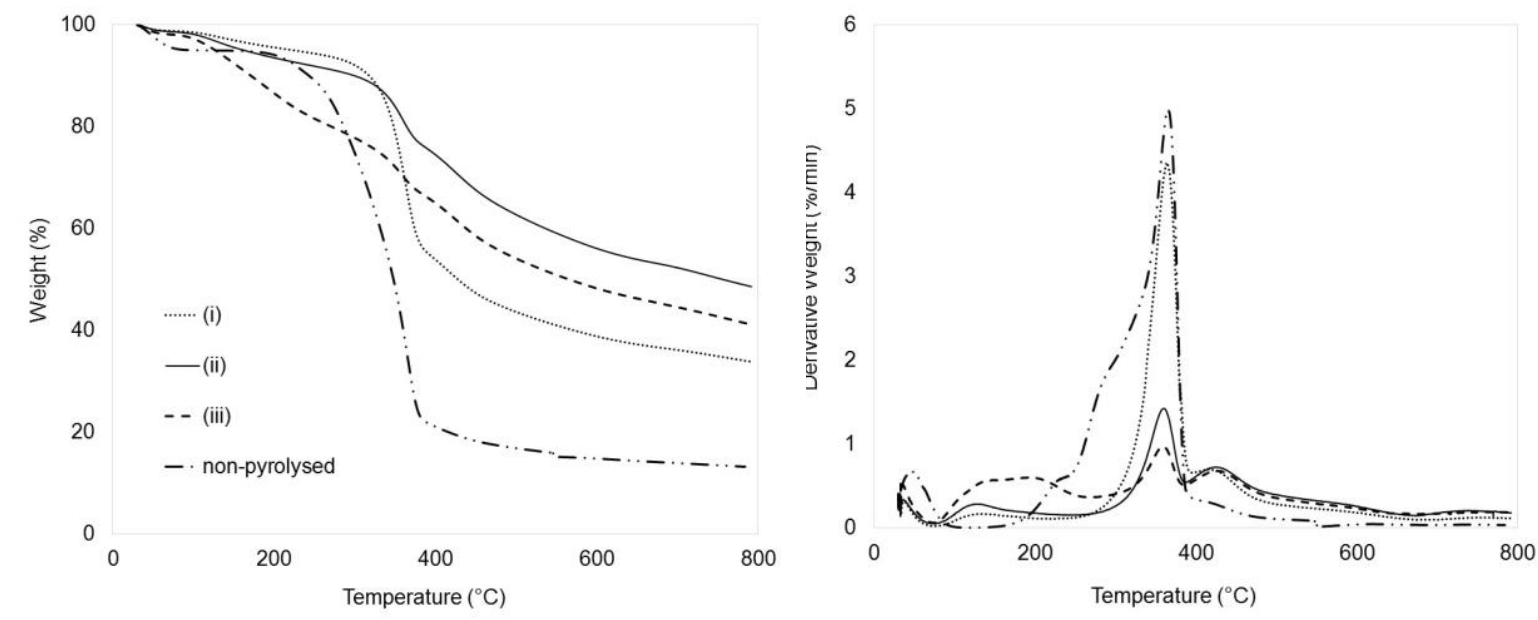

Figure 7 - Thermogravimetric analysis of sections (i), (ii), (iii) and non-pyrolysed sycamore.

Typically, the first step of thermal degradation of any lignocellulosic material is attributed to the decomposition of hemicellulose and the initial stage of degradation of cellulose (occurring between $200^{\circ} \mathrm{C}$ and $270^{\circ} \mathrm{C}$ ), while the second step is attributed to the degradation of lignin and the final degradation of cellulose (occurring between $270^{\circ} \mathrm{C}$ and $370^{\circ} \mathrm{C}$ ) [21]. This is in agreement with the thermal profile of the non-pyrolysed sycamore shown in Figure 7, which displays significant mass loss prior to the peak at $370^{\circ} \mathrm{C}$. Sections (i), (ii) and (iii) do not exhibit the same mass loss prior to the peak, suggesting the complete decomposition of hemicellulose under pyrolysis conditions. However, the smaller peaks which are apparent at around $360^{\circ} \mathrm{C}$ infer that cellulose had almost completely decomposed during the pyrolysis process. The difference in cellulose peak size between sections i, ii and iii confirms that differing degrees of pyrolysis have taken place within the block of sycamore, but nonetheless confirms that temperatures of the order of $400^{\circ} \mathrm{C}$ have been reached during the process.

\subsection{Effect of energy input on the pyrolysis process}

The extent to which the biomass had been pyrolysed was quantified by measuring the bulk mass loss after the microwave heating experiments. As the water content is $6.4 \%$, any mass loss over and above this level indicates volatilisation due to thermal decomposition of the biomass. The mass loss can be 
used as a benchmark for liquid-product yield and be used to compare with results from previous studies using microwave heating in a gaseous inert environment. A common approach used to reconcile microwave heating variables in the absence of a reliable temperature measurement is to calculate the absorbed energy based on the power, heating time and sample mass. Figure 8 shows the effect of energy input on sycamore mass loss for the complete set of experiments carried out during this study.

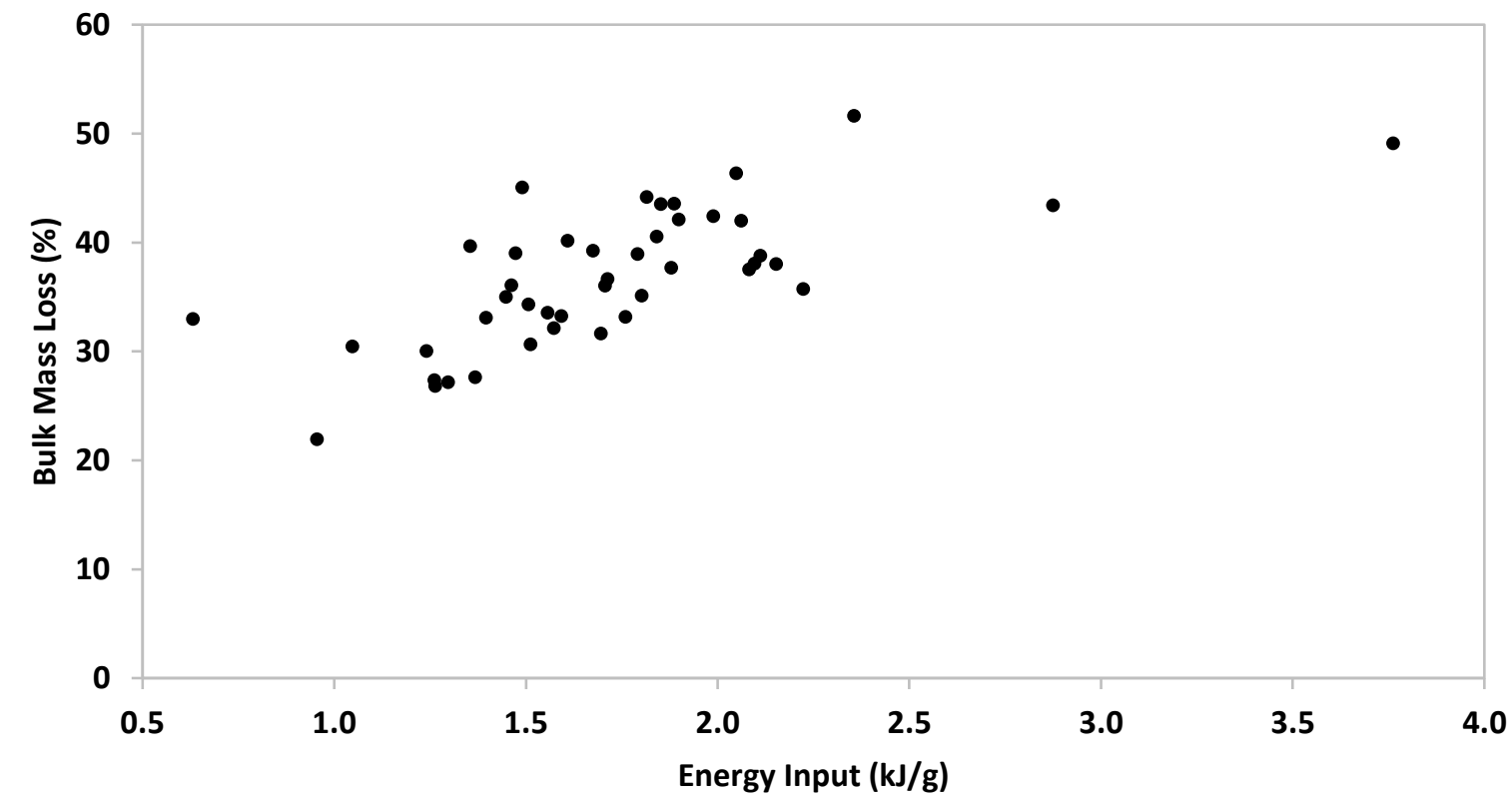

Figure 8 - Bulk mass loss of sycamore particles against energy supplied when heated within n-hexane at power levels of 1.0-1.8 $\mathrm{kW}$, heating times from 5-45 seconds.

There is a positive trend between the energy input and the mass loss for the hexane-inerted process, with higher energy input leading to greater mass loss. The maximum mass loss obtained in this study was $52 \%$, with the majority of data lying between $25-45 \%$ at energy inputs of $1.2-2.2 \mathrm{~kJ} / \mathrm{g}$. This data is comparable with data from a gas-inerted fixed bed pyrolysis process using European Larch (Larix decidua), where the majority of mass losses occurred around $1.8 \mathrm{~kJ} / \mathrm{g}$ [8]. This evidently implies that there is an energy penalty in using a hexane-inerted system and also a limit to the potential mass loss at the powers and times used. For the gas-inerted system, the mass loss reached almost $60 \%$, whereas the highest mass loss for the hexane-inerted system is $52 \%$ at $2.35 \mathrm{~kJ} / \mathrm{g}$. The lower mass loss at equivalent powers is to be expected particularly since the outer edges of the biomass sample remain in a much cooler environment (not exceeding temperatures above $68^{\circ} \mathrm{C}$ ), resulting in a larger proportion of non- 
pyrolysed material. Although the heat transfer coefficient is five times higher into the liquid than the gas the system is still conduction-limited, so changes to the convective component of heat transfer have only a minor effect on the overall heat loss and hence a minor effect on energy efficiency. The amount of solid volatilised and the energy required are similar to the values obtained with a gas-inerted system, which is remarkable given that the process takes place whilst completely submerged within a liquid and the bulk temperature maintained at $68^{\circ} \mathrm{C}$.

$\mathrm{n}$-hexane is a microwave-transparent solvent, so the biomass is heated selectively during microwave heating and n-hexane heats only by convection from the surface of the biomass. A second set of experiments were carried out using water as the inert media. Water was chosen as it is microwaveabsorbent and offers a contrast to the microwave-transparent n-hexane. Pyrolysis of the sycamore blocks was surprisingly also achieved within water and Figure 9 shows the relationship between mass loss and energy input.

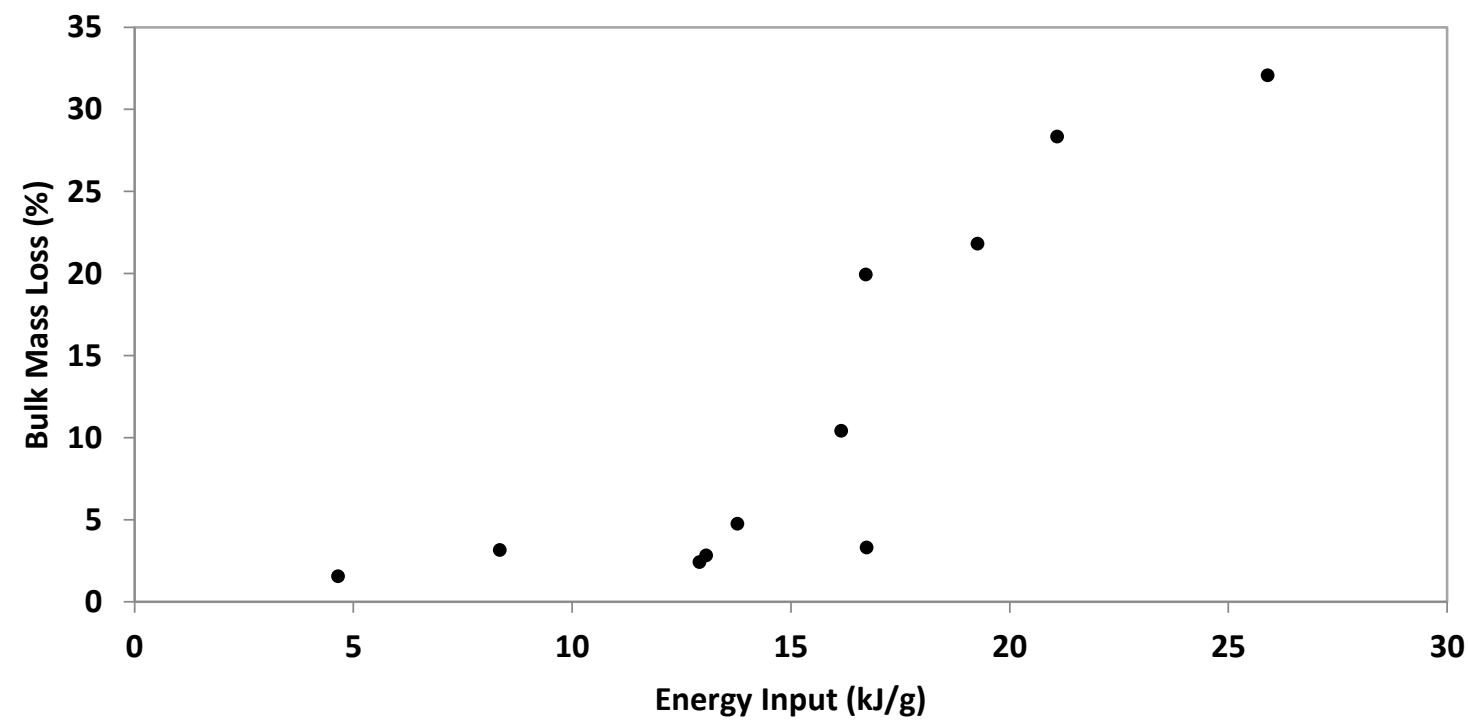

Figure 9 - Bulk mass loss of sycamore particles against energy supplied when heated within water at $1.0-1.8 \mathrm{~kW}$.

When water is used as the inert media, it appears that a threshold energy input of around $15 \mathrm{~kJ} / \mathrm{g}$ is required before any appreciable mass loss occurs. At lower energy inputs $(5-15 \mathrm{~kJ} / \mathrm{g})$ the sycamore 
remains non-pyrolysed, with the recorded mass loss corresponding to the removal of water (6.4\%) from the sycamore sample. At higher energy inputs $(15-26 \mathrm{~kJ} / \mathrm{g})$ it is possible to pyrolyse the biomass, with $32 \%$ mass loss observed at $26 \mathrm{~kJ} / \mathrm{g}$. The energy requirements are evidently higher compared to utilising $\mathrm{n}$-hexane $(\sim 2 \mathrm{~kJ} / \mathrm{g})$, which is to be somewhat expected given that water absorbs microwave energy as well as biomass. As the temperature of water increases the dielectric loss factor decreases [22], and Lee et al. [23] have demonstrated that selective heating of biomass can take place within water during microwave heating at temperatures above $50^{\circ} \mathrm{C}$ due to this phenomenon. The water temperature reached $100^{\circ} \mathrm{C}$ during the pyrolysis process as there was evidence of water boiling, so it is likely that selective heating did indeed take place and that enough microwave energy was absorbed by the biomass to allow the centre of the particle to reach temperatures sufficient to induce pyrolysis. More energy is clearly required when using water, however the data in Figures 9 and 10 indicate that the concept of pyrolysing within an inert liquid could be applied using a broad spectrum of different liquids and is not confined solely to those that are microwave transparent.

\subsection{Preliminary Product Evaluation}

Following pyrolysis in n-hexane the products were quenched in the surrounding liquid, and collected as two separate phases; an aqueous phase that was immiscible with the hexane, and an organic phase that was in solution with the hexane. The water content of the aqueous phase was $40-50 \%$ by mass, whereas the organic phase contained $<5 \%$ water. GCMS analysis of the dehydrated aqueous and organic phases was carried out, with example data shown in Table 2 and Table 3.

\begin{tabular}{|l|c|c|}
\hline \multirow{2}{*}{ Compound } & \multicolumn{2}{|c|}{ Peak Area \% } \\
\cline { 2 - 3 } & $\begin{array}{c}\text { Organic } \\
\text { phase }\end{array}$ & $\begin{array}{c}\text { Aqueous } \\
\text { phase }\end{array}$ \\
\hline Acetic acid & - & 14.0 \\
Pentanal & - & 5.1 \\
Furfural & 7.1 & 0.8 \\
2-pentanone & 4 & 1.6 \\
Guiacol & 4.5 & 0.5 \\
5-hydroxymethylfurfural & - & 1.6 \\
2-methoxy-4-vinylphenol & 6.2 & - \\
Syringol & 2 & 2 \\
Eugenol & 2 & - \\
1,2,4-trimethoxybenzene & 7.3 & 1.1
\end{tabular}




\begin{tabular}{|l|c|c|} 
Isoeugenol & 7.7 & 0.4 \\
Levoglucosan & - & 13.2 \\
\hline
\end{tabular}

Table 2 - Selected major compounds identified in organic and aqueous phases, and their corresponding abundance based on peak area

\begin{tabular}{|l|c|c|}
\hline \multirow{2}{*}{ Group } & \multicolumn{2}{|c|}{ Peak Area \% } \\
\cline { 2 - 3 } & $\begin{array}{c}\text { Organic } \\
\text { phase }\end{array}$ & $\begin{array}{c}\text { Aqueous } \\
\text { phase }\end{array}$ \\
\hline Acids and Esters & 5.4 & 18.7 \\
Aldehydes & 7.3 & 13.0 \\
Ketones & 22.8 & 19.2 \\
Phenols & 44.5 & 9.3 \\
Alcohols & 1.1 & 4.2 \\
Ethers & 11.3 & 9.7 \\
Sugars & 0.0 & 15.9 \\
Not Identified & 7.6 & 10.0 \\
\hline
\end{tabular}

Table 3 - Group abundance in organic and aqueous phases, based on peak area.

The liquid product partitions naturally into two phases. The aqueous phase contains all of the sugars, levoglucosan, 5-HMF, acetic acid and pentanal. Acids \& esters and aldehydes occur in both phases, but have a higher concentration in the aqueous phase. The organic phase is rich in Phenols, but contains relatively low amounts of alcohols and no sugars. Compounds such as furfural, guaicol and isoeugenol are more abundant in the organic phase.

The data in Table 2 and Table 3 suggest that the produced bio-oil has not suffered from extensive degradation, as evidenced by the relatively high abundance of sugars. This supports the hypothesis that conducting pyrolysis in the presence of a cold liquid leads potentially to better quality bio-oils, however further work is needed to understand how the product quality and distribution between phases varies with yield, heating rate and solvent-type. Different solvents can be chosen based on a) their boiling point and hence the resultant bulk process temperature; b) their ability to separate aqueous/organic phases and partition specific compounds within the bio-oil; c) their ability to be separated and recycled within the process; d) their ability to interact chemically with the pyrolysis products. Different process configurations will be possible depending on the solvent used; for example the hexane used in this study will allow instant and effective separation of the aqueous phase, but is likely to require a stripping process to purify the phenolic product and recycle the hexane within the pyrolysis process. The choice of solvent will therefore have a significant impact on the overall process configuration and capital cost. 
Further work is needed to better understand both the opportunities and costs of conducting microwave pyrolysis within a cold liquid environment, however this work shows that the physical principle is viable and the product quality is maintained.

\section{CONCLUSIONS}

It is shown for the first time that microwave pyrolysis of biomass can be achieved at atmospheric pressure when conventional liquid solvents are used as the inert media. The volumetric nature of microwave heating coupled with the low thermal conductivity of biomass gives rise to internal thermal gradients that allow pyrolysis to take place within the biomass despite its surface being maintained $<100^{\circ} \mathrm{C}$ due to the presence of a liquid. When a microwave transparent solvent is used the energy requirements for the liquid-phase pyrolysis process are around $2 \mathrm{~kJ} / \mathrm{g}$, which is comparable with conventional nitrogen-based systems. The ability to transform biomass at atmospheric pressure and low bulk temperatures presents a significant opportunity for utilisation of sustainable feedstocks. The liquid phase offers a potential route to higher grade products due to the low bulk temperature, and for the first time offers a robust and scalable route to use microwave heating within pyrolysis applications. Further work will aim to assess the capability of this new process concept by isolating the produced bio-oils and comparing the chemistry with gas-phase microwave processes and conventional pyrolysis techniques.

\section{ACKNOWLEDGMENTS}

The authors would like to thank Mr Richard Meehan and Mr Joseph Meehan for their technical assistance in the building of the microwave rig used in these trials. Scholarship funding was awarded

to Benjamin J. Shepherd from the EPRSC Centre for Doctoral Training in Sustainable Chemistry (University of Nottingham). 


\section{REFERENCES}

1 A. Bridgwater, D. Meier and D. Radlein, An overview of fast pyrolysis of biomass. Organic geochemistry. 30(12) (1999) 1479-1493

2 T.P. Vispute, H. Zhang, A. Sanna, R. Xiao and G.W. Huber. Renewable chemical commodity feedstocks from integrated catalytic processing of pyrolysis oils. Science, 330(6008) (2010) 12221227

3 L.S. Esteban and J.E. Carrasco, Evaluation of different strategies for pulverization of forest biomasses. Powder technology. 166(3) (2006) 139-151

4 R.J. Meredith. Engineers' handbook of industrial microwave heating. IEEE, London 1998.

5 J.P. Robinson, S.W. Kingman, R. Barranco, C.E. Snape and H. Al-Sayegh. Microwave pyrolysis of wood pellets. Industrial \& Engineering Chemistry Research. 49(2) (2009) 459-463

6 D.V. Suriapparao and R. Vinu, Bio-oil production via catalytic microwave pyrolysis of model municipal solid waste component mixtures. RSC Advances. 5(71) (2015) 57619-57631

7 X. Zhang, H. Lei, L. Wang, L. Zhu, Y. Wei, Y. Liu, G. Yadavalli and D. Yan. Renewable gasolinerange aromatics and hydrogen-enriched fuel gas from biomass via catalytic microwave-induced pyrolysis. Green Chemistry. 17(7) (2015) 4029-4036.

8 J. Robinson, C. Dodds, A. Stavrinides, S. Kingman, J. Katrib, Z. Wu, J. Medrano and R. Overend. Microwave pyrolysis of biomass: control of process parameters for high pyrolysis oil yields and enhanced oil quality. Energy \& Fuels. 29(3) (2015) 1701-1709.

9 D. Beneroso, T. Monti, E.T. Kostas and J. Robinson. Microwave pyrolysis of biomass for bio-oil production: Scalable processing concepts. Chemical Engineering Journal. 316 (2017) 481-498.

10 M. Adam, D. Beneroso, J. Katrib, S. Kingman and J.P. Robinson. Microwave fluidized bed for biomass pyrolysis. Part I: Process design. Biofuels, Bioproducts and Biorefining. 11(4) (2017) 601612.

11 M. Adam, D. Beneroso, J. Katrib, S. Kingman and J.P. Robinson. Microwave fluidized bed for biomass pyrolysis. Part II: Effect of process parameters. Biofuels, Bioproducts and Biorefining. 11(4) (2017) 613-624.

12 J.-K. Xu, J.-H. Chen, and R.-C. Sun, Hydrothermal microwave valorization of eucalyptus using acidic ionic liquid as catalyst toward a green biorefinery scenario. Bioresource technology. 193 (2015) 119-127.

13 R.K. Garlapalli, B. Wirth, and M.T. Reza, Pyrolysis of hydrochar from digestate: Effect of hydrothermal carbonization and pyrolysis temperatures on pyrochar formation. Bioresource technology. 220 (2016) 168-174.

14 Z. Lu, Z. Wu, L. Fan, H. Zhang, D. Zheng and S. Wang. Rapid and solvent-saving liquefaction of woody biomass using microwave-ultrasonic assisted technology. Bioresource Technology 199 (2016) 423-426

15 G. Li, C. Hse and T. Qin. Wood liquefaction with phenol by microwave heating and FTIR evaluation. Journal of Forestry Research 26(4) (2015) 1043-1048

16 N. Hermiz, J.B. Hasted, and C. Rosenberg, Microwave dielectric losses of n-alkanes. Journal of the Chemical Society, Faraday Transactions 2: Molecular and Chemical Physics. 78(1) (1982) 147161.

17 W.M. Haynes, CRC handbook of chemistry and physics. CRC press, Boca Raton. 2014:

18 R.H. Perry, C. Chilton, and S. Kirkpatrick, Chemical engineers handbook. McGraw Hill, New York 1999. 
19 E. Lester, M. Gong, and A. Thompson, A method for source apportionment in biomass/coal blends using thermogravimetric analysis. Journal of analytical and applied pyrolysis. 80(1) (2007) 111117.

20 D. Fardad and N. Ladommatos, Evaporation of hydrocarbon compounds, including gasoline and diesel fuel, on heated metal surfaces. Proceedings of the Institution of Mechanical Engineers, Part D: Journal of Automobile Engineering. 213(6) (1999) 625-645.

21 H. Yang, R. Yan, H. Chen, D.-H. Lee and C. Zheng. Characteristics of hemicellulose, cellulose and lignin pyrolysis. Fuel. 86(12-13) (2007) 1781-1788.

22 W. Ellison, K. Lamkaouchi, and J.-M. Moreau, Water: a dielectric reference. Journal of Molecular Liquids. 68(2-3) (1996) 171-279.

23 C.-S. Lee, E. Binner, C. Winkworth-Smith, R. John, R. Gomes and J. Robinson. Enhancing natural product extraction and mass transfer using selective microwave heating. Chemical Engineering Science 149 (2016) 97-103 